\title{
A Passive Control Method for Single Joint Exoskeleton Based on Hill Model
}

\author{
Han $\mathrm{Wu}^{1, a}$, Qing $\mathrm{Wei}^{2, \mathrm{~b}}$ and Jian Wang ${ }^{3, \mathrm{c}}$ \\ 1, 2,3 College of Mechanical Engineering and Automation, National University of Defense Technology, \\ Changsha, China \\ amoran_hansir@163.com, bwien1999@163.com, cjianwang12@nudt.edu.cn
}

Keywords: Exoskeleton Robot, Hill Model, Passive Control, Sliding Mode Control.

\begin{abstract}
Recent years, exoskeleton robot has become the focus of fields of medical rehabilitation, military, aerospace, etc. But there still exist some hardnut problems in the design of mechanism, the movement intention detection and the coupling control of human-machine. Passive control is mainly used for improving the motor recovery of hemiplegic patients. A method of generating bionic trajectories which are used in passive control methods is proposed. Firstly, the structure of the control system of the elbow joint is discussed. Based on the Hill model of skeletal muscle, a new position tracking passive control strategy is proposed, which has a highly similar compliance with human motion. According to the requirements of assistance and comfort, a proportional sliding mode controller is designed using feedback linearization. After that, the model of the control system is simulated, and the tracking characteristics of the angle and angular velocity are analyzed. Finally, the experimental results validate that the passive control strategy has high human similar compliance.
\end{abstract}

\section{Introduction}

Exoskeleton technology comes from the exoskeleton concept in biology. It can be considered as a new type of electromechanical integration device that developed by imitating the biological world [1]. According to different functions, exoskeleton can be divided into four categories: body support, movement assistance, protection, and information fusion [1]. Exoskeleton robot was studied since 1980s. The more successful products include BLEEX researched by DARPA [2], Hal-5 made by Cyberdyne[3] and Rewalk [4]. It can be used in medical rehabilitation and some devices [5,6] have already achieved the function of rehabilitation training. But limited by the material, energy, control technology, etc., at present there still has a long distance from the practical application of the robot.

Exoskeleton is driven mainly by electric, pneumatic or hydraulic cylinder. Compared with the two other cylinders, electric cylinder has advantages in stable control, fast response and high precision. And it seems to be a better choice in application of medical rehabilitation. Currently, the main control methods include preprogrammed control, electromyography (EMG) based control [7], master-slave control [8], force feedback control [9], sensitivity amplification control [10] and zero moment point (ZMP) control [11]. [12] has pointed that gait training can improve muscular strength and it is effective for stroke rehabilitation. But only preprogrammed control method is effectively used in rehabilitation. EMG based control method is difficult to recognize intentional movement and other methods have risks in patients' security. Also considering human-machine doubling, the exoskeleton robot control system is different from industrial control system. Security, flexibility, comfort and portability should also be taken into account when designing an exoskeleton.

The research concentrated on the rehabilitation treatment of patients who are affected by stroke or hemiplegia, by using a simple tunable pulse input rather than a preprogrammed signal which is complicated. As far as we know, the preprogrammed signal is usually obtained by recording movement of normal human. The signal can't be easily altered and may not be suitable for different patients. What's more a storage device is needed for recording movement data. However through the method proposed a human similar movement trajectory can be easily generated. The bionic trajectory is more come near to the real motion trajectory of human, hence the patients can receive 
better recovery. Moreover, the control system is adjustable to different people just by modifying four parameters.

\section{Control System}

Exoskeleton control system has a characteristic of man in loop [1], that is human's body or brain participates the control and decision. While in the passive control the effect of human to robot can be ignored for patients' limbs are just tracking the movement of robots without active force input or joints angle feedback. The control system is composed of two parts: simulative muscle strength signal generator and elbow robot control loop. As shown in Fig. 1.

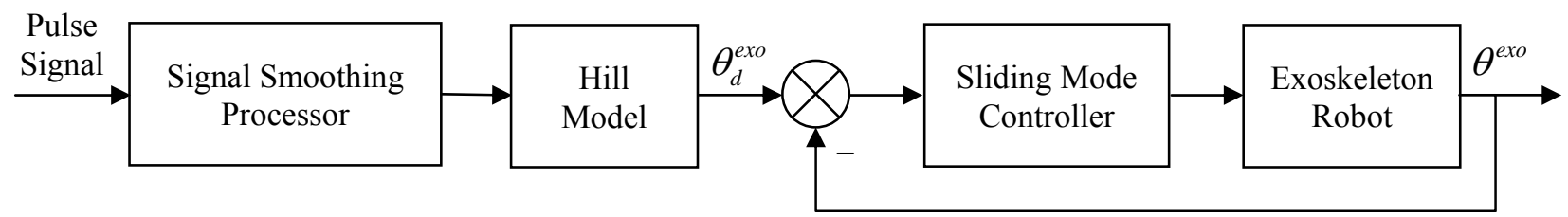

Fig. 1. Control system of elbow joint exoskeleton robot.

Hill Model of Skeletal Muscle. The main function of skeletal muscle is to output driving force for limbs' movement. The myotility is produced when the muscle is contracted actively, and the force is contacted to bone though tendon, thus joint movement is formulated. Skeletal muscle's mechanical movement is controlled by the stimulant of motor nerve [13], and the higher frequency of the stimulus the greater myotility of the muscle. An elbow joint mathematical model is built using Hill model as follows:

$$
(a+T)(v+b)=b\left(T_{0}+a\right)
$$

where $a, b$ are constants, $T_{0}$ is the myotility of muscle at the initial time $t_{0}, T$ is the myotility at time $t, v$ is the velocity of contraction.

Usually, the muscles are attached to the bone by a tendon which can't contract actively [13]. The muscle is the contraction element while the tendon, which is connected with the contraction element, is the resilience element; both of them compose the Hill model as shown in Fig. 2.
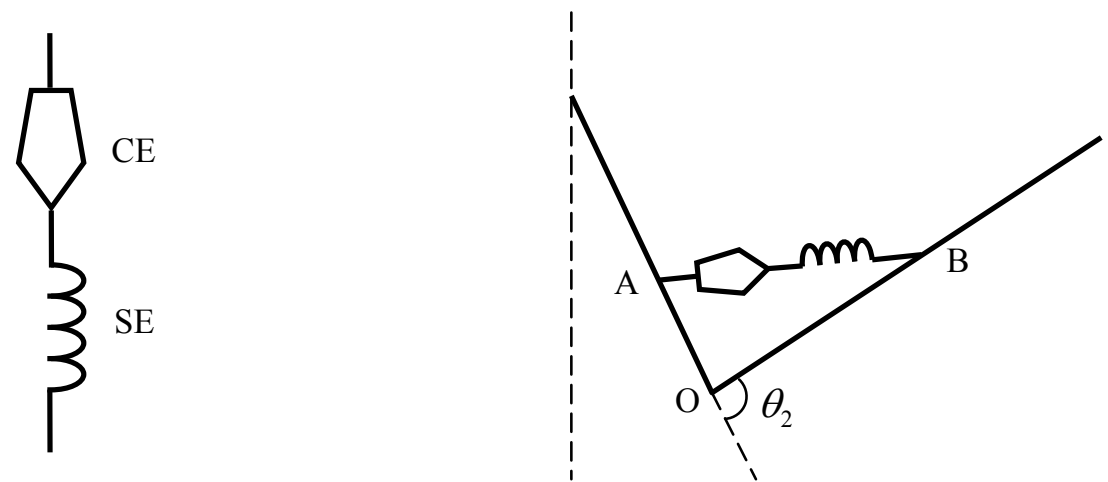

Fig. 2. Hill model of skeletal muscle.

Fig. 3. Elbow joint model.

The model proposed is based on the following two assumptions:

a. The relationship between the tension and the contraction velocity of the contraction element (CE) is determined by the instantaneous state and has nothing to do with the process of contraction.

b. In the quiescent condition, CE can expand and contract freely and is not related to the stress of muscle [1].

Human elbow joint can be modeled as a two-link linkage, as shown in Fig. 3, where $O A=d_{1}, O B=d_{2}, A B=d$. In addition, the upper arm is fixed and the forearm moves under the contraction of skeletal muscle. The skeletal muscles at elbow joint are distributed in pairs. Biceps contraction generates elbow flexion and elbow extension is the result of triceps contraction. The 
study only focuses on the motion features of biceps contraction for the similar characteristics of skeletal muscle.

The Hill equation can be expressed in the form

$$
v=\frac{b\left(T_{0}+a\right)}{a+T}-b
$$

Using the cosine theorem, we obtain

$d_{1}^{2}+d_{2}^{2}-2 d_{1} d_{2} \cos \left(\pi-\theta_{2}\right)=d^{2}$

In order to obtain the velocity of point $B$, Eq. 3 is differentiated

$2 d \cdot d=-2 d_{1} d_{2} \cdot \sin \theta_{2} \cdot \theta_{2}$

where

$\&=v$

According to Eq. 3, 4, and 5, the velocity of point $B$ can be represented by the follows:

$$
v=\frac{-2 d_{1} d_{2} \cdot \sin \theta_{2} \cdot \theta_{2}}{2 \sqrt{d_{1}^{2}+d_{2}^{2}+2 d_{1} d_{2} \cos \theta_{2}}}
$$

Substituting Eq. 6 in Eq. 2, the relationship between myotility $T$ and angle $\theta_{2}$ is obtained:

$$
T=\frac{b\left(T_{0}+a\right)}{\frac{-2 d_{1} d_{2} \cdot \sin \theta_{2} \cdot \theta_{2}}{2 \sqrt{d_{1}^{2}+d_{2}^{2}+2 d_{1} d_{2} \cos \theta_{2}}}+b}-a
$$

Muscle Strength Signal Generator. Notice that in the Hill model the myotility $T$ can't be changed steeply, which is one of the characteristics of muscle. However the control system's input is intended to be a pulsing signal in order to simplify the system. Thus a signal smoothing processor is needed. The response time of skeletal muscle contraction after a nerve stimulation is about $150 \mathrm{~ms}$ and the peak value occurs at about 30 percent [6]. The response process should be considered in the smoothing processor design.

Elbow joint has two degrees of freedom: flexion and extension, forearm pronation and supination. Only elbow flexion is considered. The range of elbow flexion is $\left(-140^{\circ} \sim-146^{\circ}\right) \sim\left(0^{\circ}\right)$ [1]. A pulse with $20 \mathrm{~ms}$ width, whose amplitude is adjustable, is used as the input. According to the constraints proposed a three order transfer function is chosen as the smoothing processor:

$$
G(s)=\frac{400}{s^{3}+15.5 s^{2}+105 s+300}
$$

The muscle strength signal generated through the processor is not same as but similar to the real myotility, which has light influence on the whole system.

Elbow Exoskeleton Robot Model. The robot model can also be simplified as a two-link linkage. As Fig. 4 shows, a DC motor is used as the actuator. Suppose that the output torque of motor drives forearm bar directly without any resistance. Using Newton-Euler method, the dynamic equation is obtained: 


$$
\tau-m_{2} g \frac{l_{2}}{2} \cos \left(\theta_{1}+\theta_{2}-\frac{\pi}{2}\right)=I \times \varepsilon
$$

where $\varepsilon=\frac{d \omega}{d t}=\frac{d^{2} \theta_{2}}{d t^{2}}, I=\frac{1}{3} m_{2} l_{2}^{2}$.

As for the controller, a sliding mode controller with a parallel integrator is used. The role of integrator is to eliminate the steady state error, which will have a great impact on human security. The controller structure is shown in Fig. 5.

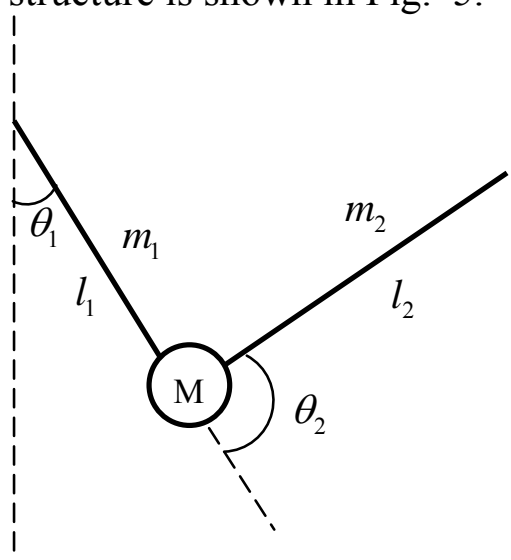

Fig. 4. Elbow exoskeleton model.

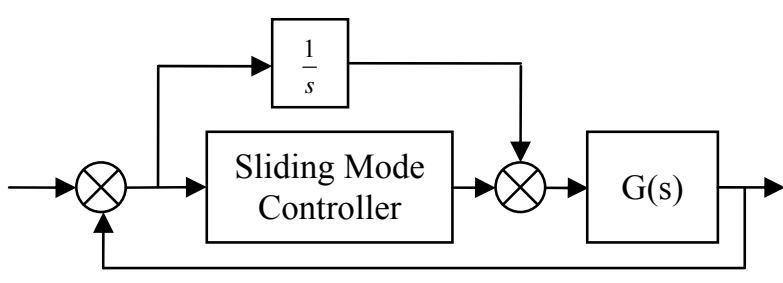

Fig. 5. Control loop of the elbow exoskeleton.

Angle error and angular velocity error of the control system are taken as the state variables. The output of the controller is

$$
u=\left(k_{1}|e|+k_{2} \mid \propto\right) \cdot \operatorname{sgn}(s)+k_{3} \int_{0}^{\infty} e d t
$$

where $s$ is switching function, $e$ is angle error, $\&$ is velocity error, $k_{1}, k_{2}$ and $k_{3}$ are constants.

$$
S=c_{1} e+c_{2} \&
$$

Thus, the whole elbow robotic exoskeleton control system is obtained.

\section{Experiments}

In order to verify the human similar compliance of the strategy proposed, four groups of joint moving data was collected through indoor experiments. As the Fig. 6, 7 shows, the device used to collect movement data is a dynamic capture product made by Motion Analysis Corporation. It can capture the relative position information of the marking points in 3D space accurately with an error about $0.1 \mathrm{~mm}$. In the experiment, the experimenter's shoulder, elbow and wrist joints are fixed with a marked point which can be detected by the device, and the elbow joint moves from fully extending to complete bending without any load. And 9 sets of data are collected in the experiment, as shown in Fig. 8 and Fig. 9. Each set of data contains a whole movement of elbow joint.

The second group data which is encircled by rectangular box in Fig. 8 and Fig. 9 is selected randomly to verify the strategy proposed. The range and continue time of the movement are taken as the initial condition of the model. According to the experimenter's arm structure the parameters of skeletal muscle model can be confirmed, as shown in Table 1 . Notice that $d_{1}$ and $d_{2}$ are the approximate distance from tendon to elbow joint rather than the length of limbs. Given a pulse signal with amplitude of 100 and width of $20 \mathrm{~ms}$, the trajectory generated is shown in Fig. 10, 11. 


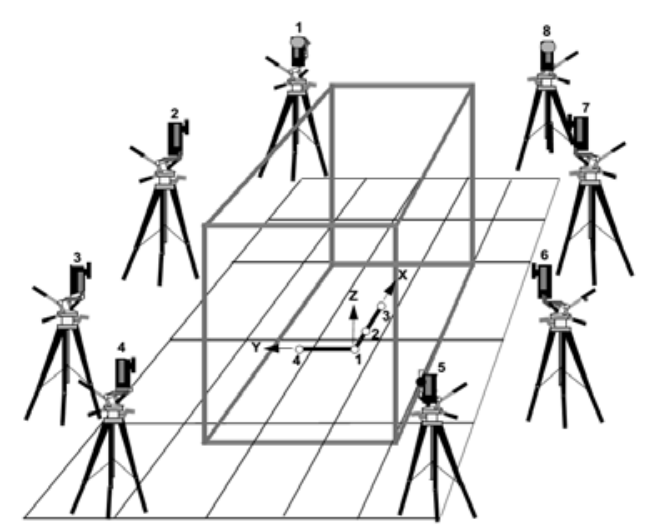

Fig. 6. Dynamic capture device.

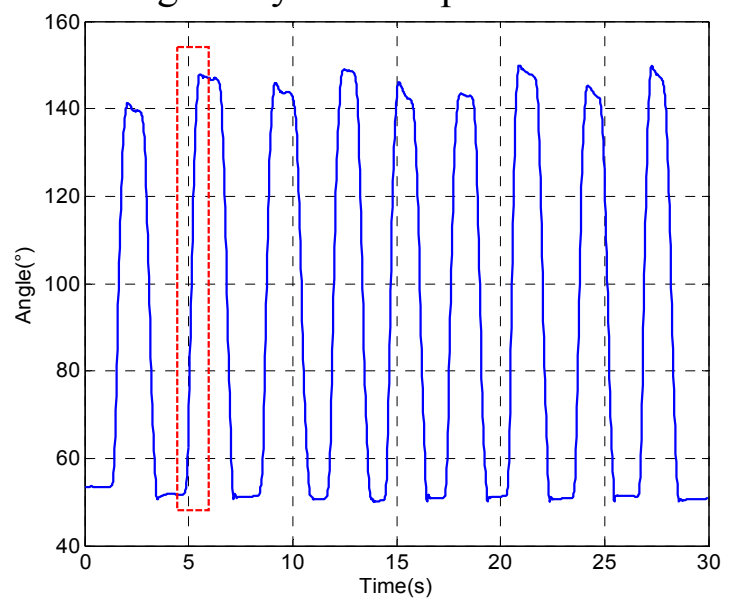

Fig. 8. Elbow joint angle.

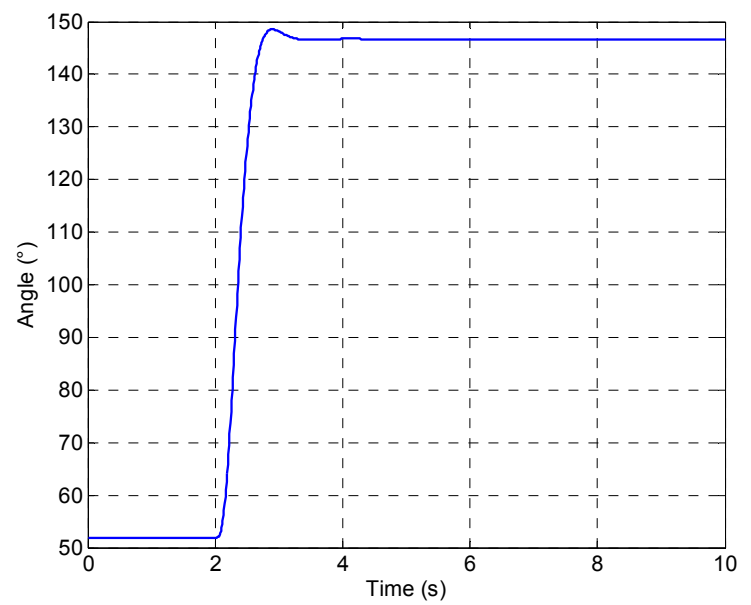

Fig. 10. Angle of the trajectory generated. Fig. 11. Angular velocity of the trajectory generated.

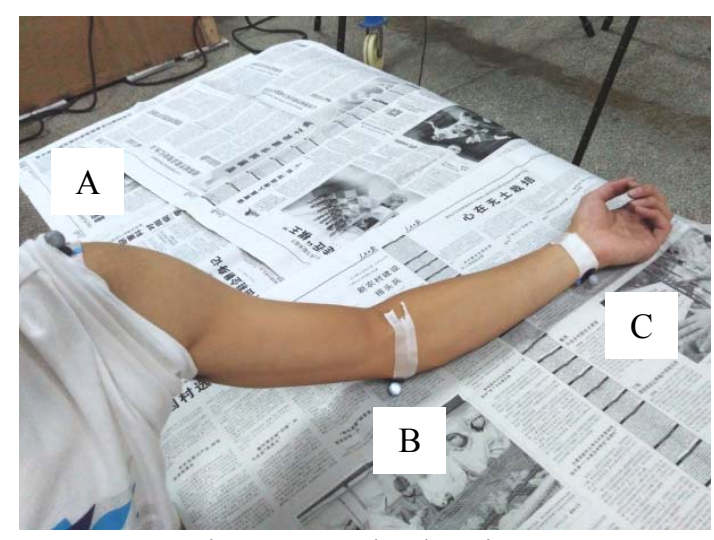

Fig. 7. Marked points.

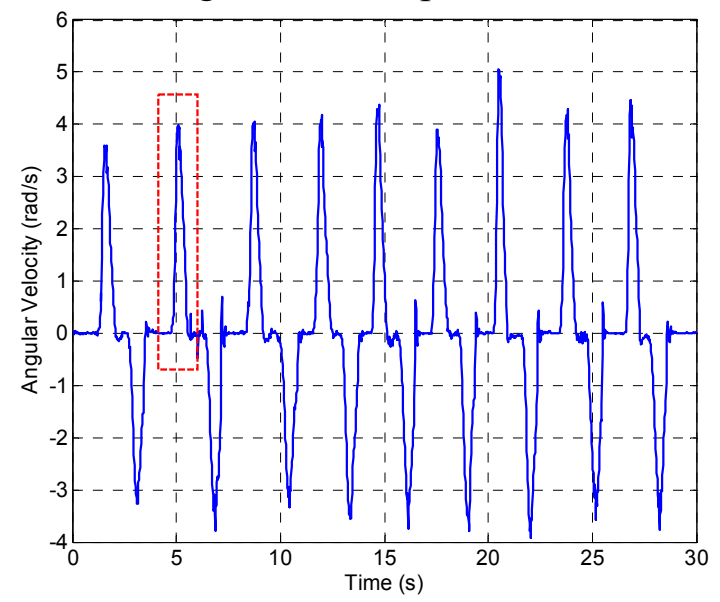

Fig. 9. Elbow joint angular velocity.

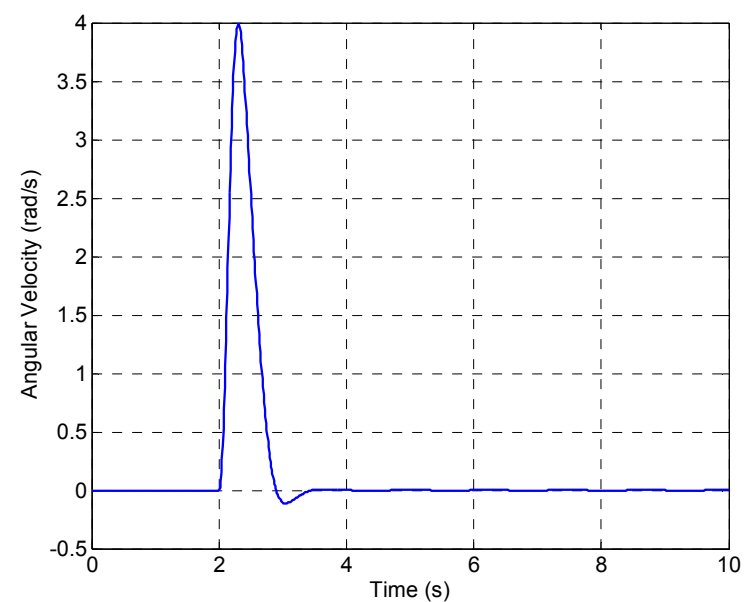

Table 1. Parameter Values.

\begin{tabular}{c|c}
\hline \hline$a$ & -318.71 \\
\hline$b$ & -0.05 \\
\hline$T_{0}$ & $0[\mathrm{~N}]$ \\
\hline$d_{1}$ & $0.2[\mathrm{~m}]$ \\
\hline$d_{2}$ & $0.01[\mathrm{~m}]$ \\
\hline$\theta_{2 \_ \text {start }}$ & $52\left[^{\circ}\right]$ \\
\hline$\theta_{2 \_ \text {end }}$ & $147\left[^{\circ}\right]$ \\
\hline \hline
\end{tabular}


The time of experiment data and output of the control system model are normalized as Fig. 12, 13 shows. The approximation of the two curves is pretty good. On the basis of the difference of shrinkage speed, muscle contraction progress can be divided into three phases: hold phase, acceleration phase and decelerating phase. The error of joint angle is below $0.035 \mathrm{rad}$ and the error of angular velocity is less than $0.15 \mathrm{rad} / \mathrm{s}$ except tow discrete points which may be caused by measurement error. The phase diagrams of experiment data and model output are shown in Fig. 14, the model output approximate to the data of experiments except two discrete points which may be caused by measurement error.

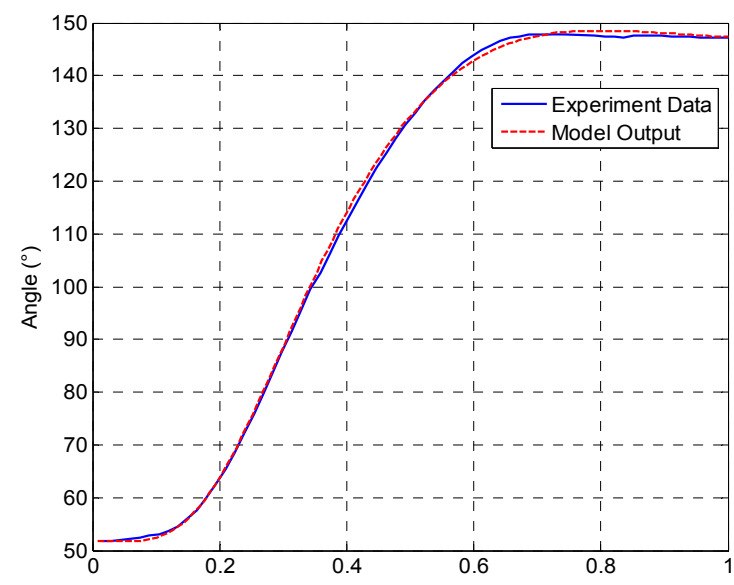

Fig. 12. Comparison of angle.

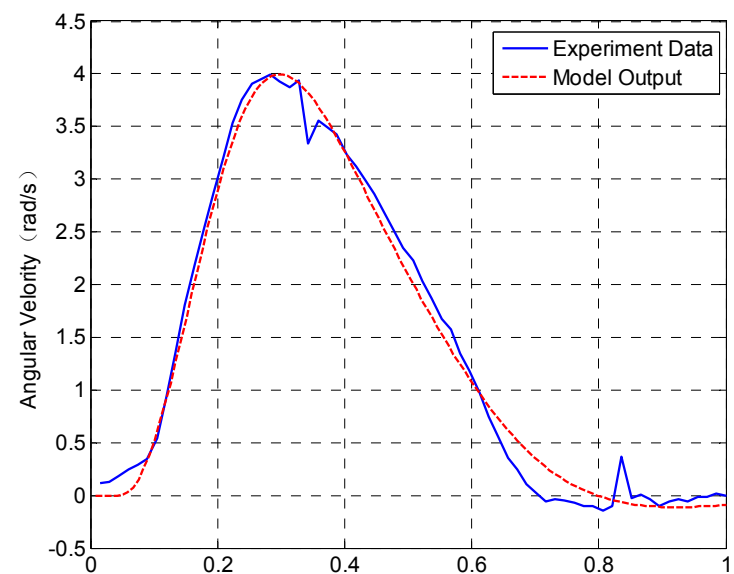

Fig. 13. Comparison of angular velocity.

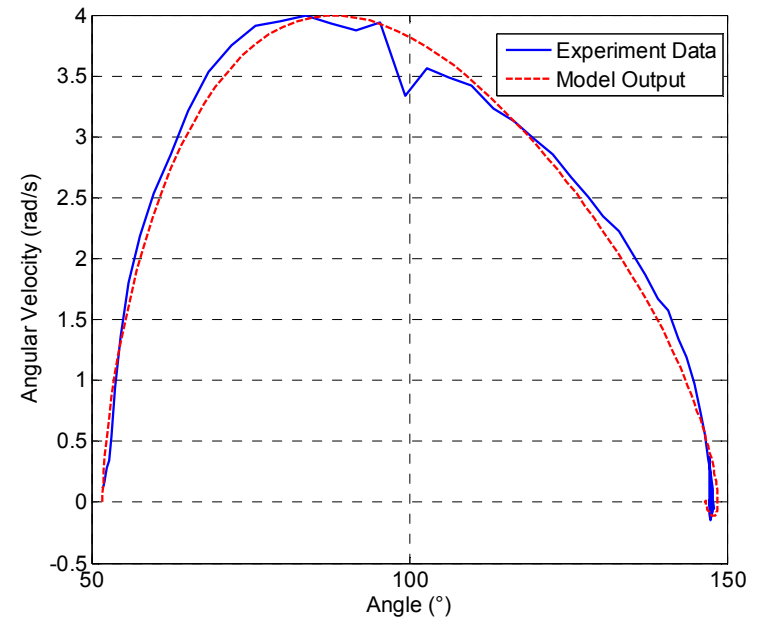

Fig. 14. Comparison of phase diagrams.

\section{Conclusions}

The study builds an elbow model and an elbow robotic exoskeleton model based on Hill model. Based on the model a passive rehabilitation training method is proposed and a sliding mode controller is designed to simulate the control system. Through experiments the biomimetic of the passive control strategy is verified.

The strategy can be used not only elbow joints but also any other body joints for the similarity of skeletal muscle. Passive training is helpful for the patients with stroke or hemiplegia and it's the main method to promote functional recovery in these subjects [14]. Traditional training methods require skilled therapists or clinicians, which is relatively expensive. Using an exoskeleton robot to conduct the training progress can release therapists and receive more effective rehabilitation. The method proposed generates motion trajectory independently and can be adjusted freely until patients feel comfortable. It has been shown that intensive and repetitive training helps patients pick up motor skills [15]. 
Besides the simple input advantage of the strategy, the system can also be used in active control mode with sEMG or other sensors. Faint signal of patients' muscle which is too weak to do normally activity can be picked up and be used as the input of the control system.

For further research, a single joint exoskeleton robot should be developed to practice the strategy above. It's also necessary to conduct experiments on some end-users in order to evaluate the effectiveness of rehabilitation.

\section{References}

[1] Jiafan Zhang, Intelligence system of robotic exoskeleton [M]. Beijing: Science Press, 2011.

[2] Kazerooni H, Racine J L, Huang L, et al. Hybrid Control of the Berkeley Lower Extremity Exoskeleton (BLEEX) [J]. International Journal of Robotics Research, 2006, 25(5-6):561-573.

[3] Nilsson A, Vreede K S, Häglund V, et al. Gait training early after stroke with a new exoskeletonthe hybrid assistive limb: a study of safety and feasibility [J]. Journal of Neuroengineering \& Rehabilitation, 2014, 11(1):1-11.

[4] Gabi Z, Harold W, Manuel Z, et al. Safety and tolerance of the ReWalk ${ }^{\mathrm{TM}}$ exoskeleton suit for ambulation by people with complete spinal cord injury: a pilot study [J]. Journal of Spinal Cord Medicine, 2012, 35(2):96-101(6).

[5] Emilia Ambrosini, Simona Ferrante, Mauro Rossini, Franco Molteni, Margit Gföhler, Werner Reichenfelser, Alexander Duschau-Wicke, Giancarlo Ferrigno and Alessandra Pedrocchi (2014). Functional and usability assessment of a robotic exoskeleton arm to support daily life. Robotica, 32, pp. 1213-1224.

[6] Cristian Coplilusi, Marco Ceccarelli and Giuseppe Carbone (2015). Design and numerical characterization of a new leg exoskeleton for motion assistance. Robotica, 33, pp. 1147-1162.

[7] Real-time controller for foot-drop correction by using surface electromyography sensor.

[8] Development of a Hand-Assist Robot With Multi-Degrees-of-Freedom for Rehabilitation Therapy.

[9] Exoskeleton arm with force feedback for robot bilateral teleoperation.

[10] Steger; John Ryan. A design and control methodology for human exoskeletons [D]. University of California, 2006.

[11]M. VUKOBRATOVIC, V.POTKONJAK and S. TZAFESTAS. Human and Humanoid Dynamics. Journal of Intelligent and Robotic Systems 41: 65-84, 2004.

[12]Lau KW, Mak MK. Speed-dependent treadmill training is effective to improve gait and balance performance in patients with sub-acute stroke. J Rehabil Med. 2011; 43:709-13.

[13] Margareta Nordin.Basic Biomechanics of the Musculoskeletal System [M]. Beijing: Ren Wei Press, 2008.

[14]G. E. Gresham, D. Alexander, D. S. Bishop, C. Giuliani, G. Goldberg, A. Holland, M. Kelly-Hayes, R. T. Linn, E. J. Roth, W. B. Stason and C. A. Trombly, "American Heart Association Prevention Conference. IV. Prevention and rehabilitation of stroke. Rehabilitation," Stroke 28(7), 1522-1526(1997).

[15]H. C. Huang, K. C. Chung, D. C. Lai and S. F. Sung, "The impact of timing and dose of rehabilitation delivery on functional recovery of stroke patients," J. Chin. Med. Assoc. 72(5), 257-264(2009). 